\title{
Critical point in the strong field magnetoresistance of a normal conductor/perfect insulator/perfect conductor composite with a random columnar microstructure
}

\author{
David J. Bergman \\ School of Physics and Astronomy, Raymond and Beverly Sackler Faculty of Exact Sciences \\ Tel Aviv University, Tel Aviv 69978, Israel \\ and \\ Department of Physics, The Ohio State University, Columbus, OH 43210-1106
}

(November 13, 2018)

\begin{abstract}
A recently developed self-consistent effective medium approximation, for composites with a columnar microstructure, is applied to such a three-constituent mixture of isotropic normal conductor, perfect insulator, and perfect conductor, where a strong magnetic field $\mathbf{B}$ is present in the plane perpendicular to the columnar axis. When the insulating and perfectly conducting constituents do not percolate in that plane, the microstructure-induced in-plane magnetoresistance is found to saturate for large $\mathbf{B}$, if the volume fraction of the perfect conductor $p_{S}$ is greater than that of the perfect insulator $p_{I}$. By contrast, if $p_{S}<p_{I}$, that magnetoresistance keeps increasing as $\mathbf{B}^{2}$ without ever saturating. This abrupt change in the macroscopic response, which occurs when $p_{S}=p_{I}$, is a critical point, with the associated critical exponents and scaling behavior that are characteristic of such points. The physical reasons for the singular behavior of the macroscopic response are discussed. A new type of percolation process is apparently involved in this phenomenon.
\end{abstract}

Theoretical and experimental studies, performed since 1993, have shown that the macroscopic electrical response of three-dimensional (3D) composites with a twodimensional (2D) or columnar microstructure can exhibit surprising forms of behavior when subject to a strong magnetic field $\mathbf{B}$. For example, when a periodic array of parallel cylindrical holes is etched into an otherwise homogeneous free-electron-conductor host, the system exhibits a strong dependence of the macroscopic or bulk effective resistivity on both the magnitude and the direction of $\mathbf{B}$, when $\mathbf{B}$ and the average current density $\langle\mathbf{J}\rangle$ both lie in the plane perpendicular to the columnar axis. This occurs whenever the Hall-to-Ohmic resistivity ratio of the conducting host, $H \equiv \rho_{\text {Hall }} / \rho_{\text {Ohmic }}=\mu|\mathbf{B}|=\omega_{c} \tau$, (here $\mu$ is the Hall mobility, $\omega_{c}$ is the cyclotron frequency, and $\tau$ is the conductivity relaxation time) is greater than 1 , and appears even if the arrat has a high point symmetry, e.g., square or triangular. $\mathrm{A}$ A strongly anisotropic magnetoresistance was also found in calculations on a periodic columnar array of perfectly conducting inclusions embedded in a similar host. 3 More recently, composites with a disordered columnar microstructure were considered, using an appropriate modification of the Bruggeman self-consistent-effective-medium-approximation. In such a system, if the pure constituents have an isotropic transport behavior and the microstructure is isotropic in the plane perpendicular to the columnar axis, and if $\mathbf{B}$ lies in that plane, then both the longitudinal and the in-plane-transverse components of the bulk effective resistivity tensor $\hat{\rho}_{e}$, denoted by $\rho_{\|}^{(e)}$ (i.e., $\langle\mathbf{J}\rangle\|\langle\mathbf{E}\rangle\| \mathbf{B}$ ) and $\widetilde{\rho}_{\perp}^{(e)}$, (i.e., $\langle\mathbf{J}\rangle \|\langle\mathbf{E}\rangle$ and they both lie in that plane but are perpendicular to $\mathbf{B}$ ) respectively, are independent of the direction of $\mathbf{B}$ or $\langle\mathbf{J}\rangle$ in that plane. In that study, it was found that for a two-constituent metal/insulator
$(M / I)$ mixture of this kind, both of these resistivity components increase as $H^{2}$ for $|H| \gg 1$, without ever reaching saturation. This behavior is not confined to the case where $M$ represents a free-electron-conductor. All that is required is that its transport behavior be isotropic, and that the Hall-to-transverse-Ohmic resistivity ratio $H$ have a magnitude that is much greater than 1 . An experimental consequence of this nonsaturating behavior would be that the bulk effective magnetoresistivities of such an $M / I$ mixture would continue to increase as $\mathbf{B}^{2}$ when $\mathbf{B}$ is large enough so that the Ohmic resistivities of the conducting constituent are saturated but its Hall resistivity continues to increase as $\mathbf{B}$. By contrast, in a normal conductor/perfect conductor $(M / S)$ mixture of this kind, both of those resistivity components saturate at finite values when $|H| \gg 1$.

Here we consider the macroscopic response of a three-constituent columnar composite of isotropic normal conductor, perfect insulator, and perfect conductor $(M / I / S)$, where the 2D microstructure in the plane perpendicular to the columnar axis is again random, and a strong magnetic field $\mathbf{B}$ is applied in that plane.

We use the "columnar unambiguous self consistent effective medium approximation (CUSEMA)", which was developed in Ref. 4 . In this approximation, the self consistency requirement is that the in-plane components of the extra electric field $\mathbf{E}$ produced by isolated circularcylindrical inclusions of the different constituents, embedded in the fictitious uniform effective medium, vanish on average. A similar requirement is not imposed upon the columnar component of $\mathbf{E}$, which would be unmeasurable in a thin film realization of such microstructures. Also, the columnar component of $\langle\mathbf{J}\rangle$ is always assumed to vanish. These requirements lead to the following self consistencey equations (we take $x$ to be the columnar 
axis)

$$
0=\left\langle\left[\left(\hat{I}-\hat{\rho}_{e} / \hat{\rho}_{\text {inc }}\right) \cdot \hat{\gamma}_{\text {inc }}\left(\hat{\rho}_{\text {inc }}, \hat{\rho}_{e}\right) \cdot \hat{\rho}_{e}\right]_{\{y z\}}\right\rangle,
$$

where $\hat{I}$ is the unit tensor, \langle\rangle indicates an average over the different types of inclusions, while the subscript $\{y z\}$ indicates that only the $y, z$ components of the $3 \times 3$ tensor appearing in the square brackets [ ] are included in this calculation, i.e., the $2 \times 2$ matrix in the lower right corner of $\left[\left(\hat{I}-\hat{\rho}_{e} / \hat{\rho}_{\text {inc }}\right) \cdot \hat{\gamma}_{\text {inc }}\left(\hat{\rho}_{\text {inc }}, \hat{\rho}_{e}\right) \cdot \hat{\rho}_{e}\right]$. The $3 \times 3$ tensor $\hat{\gamma}_{\text {inc }}\left(\hat{\rho}_{\text {inc }}, \hat{\rho}_{e}\right)$ gives the uniform local electric field $\mathbf{E}_{\text {int }}$, which appears inside an isolated circular-cylindrical inclusion, with resistivity tensor $\hat{\rho}_{\text {inc }}$, inside the otherwise uniform effective medium host $\hat{\rho}_{e}$, when a uniform electric field $\mathbf{E}_{0}$ is applied at large distances

$$
\mathbf{E}_{\text {int }}=\hat{\gamma}_{\text {inc }} \cdot \mathbf{E}_{0} .
$$

This tensor must be calculated from $\hat{\rho}_{\text {inc }}$ and $\hat{\rho}_{e}$ - this was done for the relevant types of inclusions in the Appendix of Ref. 4 .

It is important to note that the self consistency requirements used to derive CUSEMA differ from the requirements which are used to set up the conventional two-dimensional, Bruggeman-type, self-consistent effective medium approximation (SEMA) in the $y, z$ plane of this system (see Ref. 5 for a discussion of SEMA in the presence of a magnetic field, when the conductivity tensors are nonscalar tensors). Consequently, it should come as no surprise if the results of those approximations are sometimes quite different, as shown in Ref. 6 . The great advantage of using CUSEMA is that the exact relations which $\hat{\rho}_{e}$ must satisfy as a result of the duality symmetry are satisfied automatically, whereas theycan be seriously violated in the two-dimensional SEMA. 1 -

For the resistivity tensors of the effective medium host and the three different types of inclusions we write

$$
\hat{\rho}_{e}=\rho_{M}\left(\begin{array}{ccc}
\alpha_{e} & -\beta_{e} & 0 \\
\beta_{e} & \gamma_{e} & 0 \\
0 & 0 & \lambda_{e}
\end{array}\right) ; \quad \hat{\rho}_{M}=\rho_{M}\left(\begin{array}{ccc}
1 & -H & 0 \\
H & 1 & 0 \\
0 & 0 & \nu
\end{array}\right) ;
$$

$\hat{\rho}_{I}=\rho_{I} \hat{I}, \quad \rho_{I} \gg \rho_{M} ; \quad \hat{\rho}_{S}=\rho_{S} \hat{I}, \quad \rho_{S} \ll \rho_{M}$.

These forms mean that a magnetic field $\mathbf{B}$ is applied along the $z$ axis, which is perpendicular to the columnar axis $x$.

The form assumed for $\hat{\rho}_{M}$ means that the $M$ constituent exhibits isotropic transport behavior even when $\mathbf{B}$ is very strong, and does not rule out a dependence of its transverse and longitudinal Ohmic resistivities, $\rho_{M}$ and $\nu \rho_{M}$, and also of its Hall coefficient $H \rho_{M} /|\mathbf{B}|$, upon the magnitude of $\mathbf{B}$. However, it does rule out the possibility of clean single crystal inclusions of a transition metal like Copper, where the resistivities become very sensitive to the crystal orientation with respect to $\mathbf{B}$ and $\mathbf{J}$ at strong fields and low temperatures due to the existence of open orbits on its Fermi surface, although polycrystalline inclusions of such a metal might still be allowed. By contrast, the form assumed for $\hat{\rho}_{e}$ means that we expect the bulk effective transport behavior to be at least mildly anisotropic as a result of the columnar microstructure, i.e., in general we expect to have $\alpha_{e} \neq \gamma_{e}$.

The forms assumed for $\hat{\rho}_{I}$ and $\hat{\rho}_{S}$ include the case where $\hat{\rho}_{I}=\infty$ and $\hat{\rho}_{S}=0$. In fact, it is only for those extreme values that a mathematical singularity will actually be found to appear in the bulk effective macroscopic response, justifying the term "critical point".

The same resistivity scale $\rho_{M}$ is used in both $\hat{\rho}_{M}$ and $\hat{\rho}_{e}$, whose in-plane components will obviously have similar magnitudes when neither the $I$ constituent nor the $S$ constituent percolates in that plane, i.e., when their volume fractions $p_{I}, p_{S}$ satisfy $p_{I}<1 / 2, p_{S}<1 / 2$. However, because there are perfectly conducting inclusions that span the system from end to end along the columnar axis $x$, therefore the components of $\hat{\rho}_{e}$ which involve that axis will all vanish. More precisely, we will have $\alpha_{e}=O\left(\rho_{S} / \rho_{M}\right), \beta_{e}=O\left(\rho_{S} / \rho_{M}\right)$ as long as the volume fraction $p_{S}$ of the $S$ constituent is nonzero.

The $\hat{\gamma}_{\text {inc }}\left(\hat{\rho}_{\text {inc }}, \hat{\rho}_{e}\right)$ matrices for the three types of inclusions which must be considered are 4 (we omit the subscript $e$ from $\alpha_{e}, \beta_{e}, \gamma_{e}$, and $\lambda_{e}$ in these expressions, in order to save space)

$$
\begin{aligned}
& \hat{\gamma}_{\mathrm{inc}}\left(\hat{\rho}_{M}, \hat{\rho}_{e}\right)= \\
& =\left(\begin{array}{ccc}
1 & 0 & 0 \\
\frac{-\frac{\beta}{\alpha}+\frac{\gamma H}{1+H^{2}}}{\left(\frac{\gamma}{\lambda}\right)^{1 / 2}+\frac{\gamma}{1+H^{2}}} & \frac{1+\left(\frac{\gamma}{\lambda}\right)^{1 / 2}}{\left(\frac{\gamma}{\lambda}\right)^{1 / 2}+\frac{\gamma}{1+H^{2}}} & 0 \\
0 & 0 & \frac{1+\left(\frac{\gamma}{\lambda}\right)^{1 / 2}}{1+\frac{\lambda}{\nu}\left(\frac{\gamma}{\lambda}\right)^{1 / 2}}
\end{array}\right),
\end{aligned}
$$

where we omitted the term $\beta^{2} / \alpha$, which is $O\left(\rho_{S} / \rho_{M}\right) \ll$ 1 , when it appeared alongside $\gamma$, which is $O(1)$,

$$
\hat{\gamma}_{\text {inc }}\left(\hat{\rho}_{I}, \hat{\rho}_{e}\right)=\left(\begin{array}{ccc}
1 & 0 & 0 \\
\frac{-\frac{\beta}{\alpha}}{\left(\frac{\gamma}{\lambda}\right)^{1 / 2}} & \frac{1+\left(\frac{\gamma}{\lambda}\right)^{1 / 2}}{\left(\frac{\gamma}{\lambda}\right)^{1 / 2}} & 0 \\
0 & 0 & 1+\left(\frac{\gamma}{\lambda}\right)^{1 / 2}
\end{array}\right),
$$

where we also discarded $O\left(\rho_{M} / \rho_{I}\right) \ll 1$ terms, and

$$
\hat{\gamma}_{\mathrm{inc}}\left(\hat{\rho}_{S}, \hat{\rho}_{e}\right)=\left(\begin{array}{ccc}
1 & 0 & 0 \\
\frac{-\frac{\beta}{\alpha}}{\gamma \frac{\rho_{M}}{\rho_{S}}} & \frac{1+\left(\frac{\gamma}{\lambda}\right)^{1 / 2}}{\gamma \frac{\rho_{M}}{\rho_{S}}} & 0 \\
0 & 0 & \frac{1+\left(\frac{\gamma}{\lambda}\right)^{1 / 2}}{\frac{\rho_{M}}{\rho_{S}} \lambda\left(\frac{\gamma}{\lambda}\right)^{1 / 2}}
\end{array}\right),
$$

where we kept some $O\left(\rho_{S} / \rho_{M}\right) \ll 1$ terms, because those terms are subsequently multiplied by an $O\left(\rho_{M} / \rho_{S}\right) \gg 1$ term-see Eq. (何).

There will be two coupled nontrivial self-consistency equations, resulting from the $y y$ and $z z$ components of Eq. (1) (the $y z$ and $z y$ components of that equation are 
satisfied automatically due to the $2 \mathrm{D}$ isotropy of the microstructure). Thus, we get two coupled algebraic equations for the two unknown quantities $\lambda_{e} \equiv \rho_{\|}^{(e)} / \rho_{M}$ and $\gamma_{e} \equiv \widetilde{\rho}_{\perp}^{(e)} / \rho_{M}$ :

$$
\begin{aligned}
0= & \left(\frac{\gamma_{e}}{\lambda_{e}}\right)^{1 / 2}\left[\left(1-2 p_{S}\right)\left(1+H^{2}\right)-\left(1-p_{I}\right) \gamma_{e}+p_{I} \lambda_{e}\right] \\
& -\frac{\gamma_{e}}{\lambda_{e}} p_{S}\left(1+H^{2}\right)+\left(2 p_{I}-1\right) \gamma_{e}+\left(1-p_{S}\right)\left(1+H^{2}\right),
\end{aligned}
$$

$$
\left(\frac{\gamma_{e}}{\lambda_{e}}\right)^{1 / 2}=\frac{\nu p_{S}-\gamma_{e} p_{I}}{\nu\left(1-p_{S}\right)-\lambda_{e}\left(1-p_{I}\right)} .
$$

These equations can be transformed into a single polynomial equation for, say, $\lambda_{e}$. That would be an 8th order equation, which is quite complicated and which I have not been able to factorize algebraically. On the other hand, if we are interested only in the asymptotic behavior of $\lambda_{e}$ and $\gamma_{e}$ when $|H| \gg 1$, then an explicit solution can be obtained using asymptotic analysis. The results are

$$
\gamma_{e} \cong \begin{cases}H^{2} \frac{p_{I}-p_{S}}{1-2 p_{I}} \frac{1-p_{I}}{p_{I}} & \text { for } p_{I}>p_{S},\left|\left(p_{I}-p_{S}\right) H\right| \gg 1, \\ \nu \frac{1-2 p_{S}}{p_{S}-p_{I}} \frac{1-p_{S}}{p_{S}} & \text { for } p_{I}<p_{S},\left|\left(p_{I}-p_{S}\right) H\right| \gg 1, \\ \sqrt{\nu}|H| \frac{1-p_{I}}{p_{I}} & \text { for } p_{I} \gtrless p_{S},\left|\left(p_{I}-p_{S}\right) H\right| \ll 1,\end{cases}
$$

$\frac{\gamma_{e}}{\lambda_{e}} \cong\left\{\begin{array}{l}\left(\frac{1-p_{I}}{p_{I}}\right)^{2} \text { for } \gamma_{e} \gg 1 \\ \left(\frac{1-p_{S}}{p_{S}}\right)^{2} \text { for } \gamma_{e}=O(1)\end{array}\right.$

These results can be recast with the help of two scaling functions

$$
\gamma_{e} \cong \frac{\nu}{p_{I}-p_{S}} F(Z), \quad \lambda_{e} \cong \frac{\nu}{p_{I}-p_{S}} G(Z),
$$

where the scaling variable is $Z \equiv|H|\left(p_{I}-p_{S}\right) / \sqrt{\nu}$, and the scaling functions have the following limiting forms

$$
\begin{aligned}
& F(Z) \cong \begin{cases}\frac{1-p_{I}}{p_{I}} \frac{Z^{2}}{1-2 p_{I}} & \text { for } Z \gg 1, \\
-\frac{1-p_{S}}{p_{S}}\left(1-2 p_{S}\right) & \text { for } Z \ll-1, \\
\frac{1-p_{I}}{p_{I}} Z & \text { for }|Z| \ll 1,\end{cases} \\
& G(Z) \cong \begin{cases}\frac{p_{I}}{1-p_{I}} \frac{Z^{2}}{1-2 p_{I}} & \text { for } Z \gg 1, \\
-\frac{p_{S}}{1-p_{S}}\left(1-2 p_{S}\right) & \text { for } Z \ll-1, \\
\frac{p_{I}}{1-p_{I}} Z & \text { for }|Z| \ll 1 .\end{cases}
\end{aligned}
$$

Both of these functions have the qualitative form shown in Fig. 11.

Clearly, $|H|=\infty, p_{S}=p_{I}$ defines a line of critical points of the macroscopic magnetotransport of such systems: For $p_{S}>p_{I}$, both $\rho_{\|}^{(e)}$ and $\tilde{\rho}_{\perp}^{(e)}$ saturate when $|H| \rightarrow \infty$, whereas for $p_{S}<p_{I}$ they both keep increasing as $H^{2}$ for $|H| \gg 1$. As $p_{S} \rightarrow p_{I}$ from below, the coefficients of the $H^{2}$ terms tend to zero as $p_{I}-p_{S}$, while if $p_{S} \rightarrow p_{I}$ from above the saturated values of both $\rho_{\|}^{(e)}$ and $\widetilde{\rho}_{\perp}^{(e)}$ diverge as $1 /\left(p_{S}-p_{I}\right)$. When $p_{S}=p_{I}$, then these resistivity components keep increasing as $|H|$ for $|H| \gg 1$. In the vicinity of the critical line, where both $1 /|H|$ and $\left|p_{S}-p_{I}\right|$ are very small, it is easy to see that $F(Z) \cong G(Z)$ and $\gamma_{e} \cong \lambda_{e}$.

Because these results were obtained within the framework of CUSEMA, some of these behaviors are not expected to be correct in detail. We do expect that even a more accurate calculation of asymptotic behavior (i.e., $|H| \gg 1)$ will exhibit saturated behavior for $p_{I}<p_{S}$, nonsaturating $\propto H^{2}$ behavior for $p_{I}>p_{S}$, and nonsaturating $\propto|H|$ behavior for $p_{I}=p_{S}$. However, we expect that more accurate calculations will show that the critical behavior as $p_{S} \rightarrow p_{I}$ is not characterized by the simple forms $1 /\left(p_{S}-p_{I}\right)$ or $p_{I}-p_{S}$ which were obtained here, but rather by some noninteger values of the critical exponents. Such calculations are now in progress.

The behavior found in these calculations can be understood qualitatively by recalling that the in-plane $y, z$ components of $\hat{\sigma}_{M}$ are

$$
\frac{1}{\rho_{M}}\left(\begin{array}{cc}
\frac{1}{1+H^{2}} & 0 \\
0 & \frac{1}{\nu}
\end{array}\right) \text {. }
$$

For $|H| \gg 1$, this represents a very anisotropic $2 \mathrm{D}$ conductor in the $y, z$ plane, with $\rho_{M} \sigma_{M z z}=1 / \nu=O(1)$ and $\rho_{M} \sigma_{M y y} \cong 1 / H^{2} \ll 1$. In order to get from end to end of the sample, the in-plane electric current must make its way between different $S$ inclusions by flowing through the $M$ host. If there are many more $S$ inclusions than $I$ inclusions, then straight line $S$-to- $S$ trajectories can easily be found that are parallel to $z$, allowing the current to flow through the $M$ constituent only in the $z$ direction. Therefore the macroscopic conductivity will depend only on $\sigma_{M z z}$, and the macroscopic resistivity will saturate when $|H| \gg 1$. In the opposite case, when there are many more $I$ inclusions than $S$ inclusions, the current will often not be able to flow even between neighboring $S$ inclusions only along $z$, but will have to have a nonzero $y$ component in the $M$ constituent. This is the low conductivity direction, therefore the macroscopic conductivity will now be determined primarily by $\sigma_{M y y} \cong 1 /\left(\rho_{M} H^{2}\right)$. Threfore the macroscopic resistivity will not saturate, but keep increasing as $H^{2}$ forever.

As the relative proportion of the $I$ and $S$ constituents is varied, there will be a transition from saturating to nonsaturating $\propto H^{2}$ behavior, which will have to be abrupt, i.e., it will be a singular or critical point of the macroscopic response. This will be reflected by a similarly 
abrupt change in the detailed local current distribution, which will have only $z$-parallel flow lines in the $M$ constituent for values of $p_{I}$ below the transition point value, but will also have nonzero values of $J_{y}$ in that constituent when $p_{I}$ is above that value. Such a transition constitutes a new type of percolation phenomenon, which we believe deserves further study, both theoretical and experimental.

Experimental study of the critical point we have discovered could be done using a doped semiconductor film as the $M$ constituent, with a random collection of etched perpendicular holes as the $I$ constituent, and a random collection of perpendicular columnar inclusions, made of a high conductivity normal metal, playing the role of the $S$ constituent. We would like to note that extremely low temperatures or very clean single crystals would not be required in order to observe this critical point. What would be necessary is a large contrast at each stage of the following chain of inequalities

$$
\rho_{S} \ll \rho_{M} \ll H^{2} \rho_{M} \ll \rho_{I} .
$$

If Si-doped GaAs is used as the $M$ host, with a negative charge carrier density of $1.6 \times 10^{18} \mathrm{~cm}^{-3}$ and a mobility $\mu=2500 \mathrm{~cm}^{2} / \mathrm{Vs}$ at a temperature of $90 \mathrm{~K}$, as in the experiment described in Ref. 2, then a magnetic field of 40 Tesla would result in $H=-10$. Such a material would have an Ohmic resistivity of $1.6 \times 10^{-3} \Omega \mathrm{cm}$, about 1000 times greater than that of Copper. Thus, using Copper for the $S$ inclusions and etched holes for the $I$ inclusions, there should be no difficulty in satisfying all the above inequalities.

\section{ACKNOWLEDGMENTS}

This research was supported in part by grants from the US-Israel Binational Science Foundation, the Israel Science Foundation, and NSF Grant DMR 97-31511.

${ }^{1}$ D. J. Bergman and Y. M. Strelniker, Phys. Rev. B 49, 16256 (1994).

${ }^{2}$ M. Tornow, D. Weiss, K. v. Klitzing, K. Eberl, D. J. Bergman, and Y. M. Strelniker, Phys. Rev. Lett. 77, 147 (1996).

${ }^{3}$ D. J. Bergman and Y. M. Strelniker, Phys. Rev. B 59, 2180 (1999).

${ }^{4}$ D. J. Bergman and Y. M. Strelniker, Phys. Rev. B 60, 13016 (1999).

${ }^{5}$ D. Stroud, Phys. Rev. B 12, 3368 (1975).

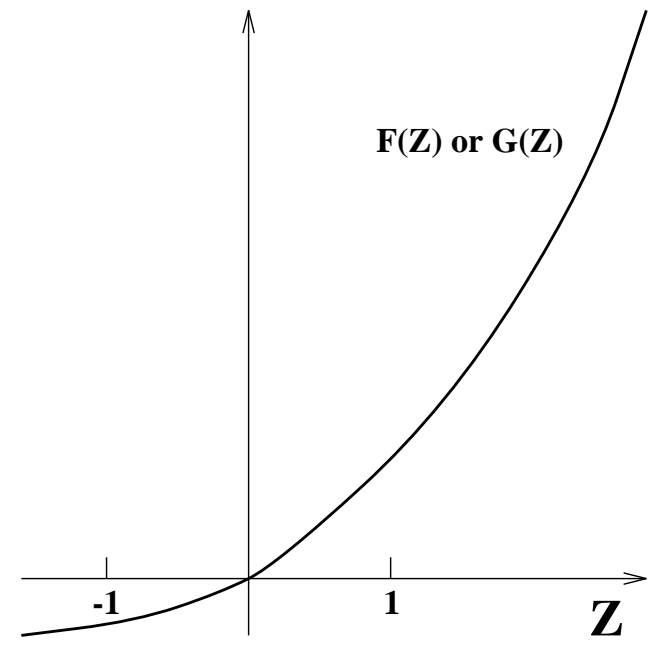

FIG. 1. Qualitative shape of the two scaling functions $F(Z)$ and $G(Z)$-see Eqs. 13) and (14). 